\title{
REVIEW
}

\section{TARGETING SWI/SNF METABOLIC VULNERABILITIES IN CANCER}

\author{
M. Soeung ${ }^{1}$, L. Perelli ${ }^{2,3}$, A. Sgambato ${ }^{3,4}$, G. Genovese ${ }^{2,5}$
}

${ }^{1}$ Department of Genomic Medicine, The University of Texas MD Anderson Cancer Center, Houston, TX, USA

${ }^{2}$ Department of Genitourinary Medical Oncology, The University of Texas MD Anderson Cancer Center, Houston, TX, USA

${ }^{3}$ Department of Translational Medicine and Surgery, Università Cattolica del Sacro Cuore, Rome, Italy

${ }^{4}$ Scientific Direction, Centro di Riferimento Oncologico della Basilicata - IRCCS-CROB, Rionero in Vulture, Potenza, Italy

${ }^{5}$ David H. Koch Center for Applied Research of Genitourinary Cancers, The University of Texas MD Anderson Cancer Center, Houston, TX, USA

\section{CORRESPONDING AUTHOR:}

\section{Giannicola Genovese}

Department of Genitourinary Medical Oncology

The University of Texas MD Anderson Cancer Center

David H. Koch Center for Applied Research of Genitourinary Cancers

1515 Holcombe Blvd

77030 Houston, TX, USA

E-mail: ggenovese@mdanderson.org

ORCID:

Doi: $10.48286 /$ aro.2021.31

History

Received: Jul 27, 2021

Accepted: Nov 29, 2021

Published: Dec 1, 2021

\section{ABSTRACT}

The SWI/SNF (Switch/Sucrose non-fermentable) complex is a key epigenetic regulator that is conserved across different species. While its functions as a gene expression regulator have been widely characterized, new evidences suggest that it can act as a fundamental modulator of metabolic pathways both in physiological and pathological processes. In this review, we summarized the most recent literature addressing molecular interactions involving members of the SWI/SNF complex and metabolic pathways. We focused on how genetic alterations of SWI/SNF subunits lead to tumorigenesis and aggressive phenotypes during cancer evolution. Finally, we highlighted metabolic vulnerabilities of cancer cells with altered SWI/SNF complex that can be exploited as future clinical targets for the treatment of advanced disease. SWI/SNF complex is a critical regulator of chromatin accessibility frequently mutated in human cancers. Mutations in the SWI/SNF complex renders cancer cells vulnerable to metabolic perturbations, that may constitute new potential oncological targets. 


\section{KEY WORDS}

SWIISNF; Metabolism; Cancer; Vulnerabilities.

\section{IMPACT STATEMENT}

SWI/SNF complex is a critical regulator of chromatin accessibility frequently mutated in human cancers. Mutations in the SWI/SNF complex renders cancer cells vulnerable to metabolic perturbations, that may constitute new potential oncological targets.

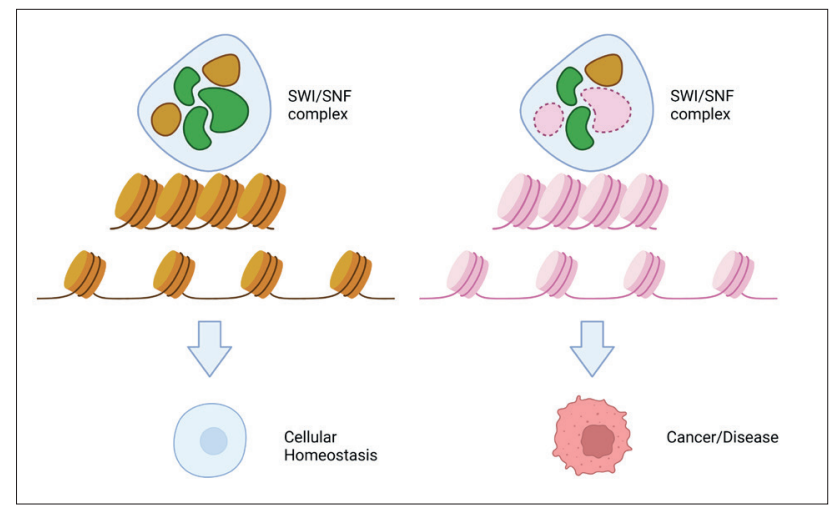

Figure 1. Illustration showing how the SWI/SNF complex plays a critical role in maintaining cellular homeostasis by modulating chromatin accessibility (left). Consequentially, SWI/SNF mutations can lead to disruptions in cellular homeostasis that lead to disease (right). Created with BioRender.com. a plethora of cellular processes to maintain homeostasis by changing the accessibility status of chromatin, thereby activating or repressing gene transcription $(1,2)$. Studies have suggested that the SWI/SNF subunits are likely tightly regulated to maintain their critical functions in establishing cellular homeostasis (3). Consequently, an imbalance in the concentration of SWI/SNF proteins can lead to diseases like cancer (figure 1). Bultman and colleagues showed that homozygous knockout of SMARCA4 (also called BRG1) in mouse models was embryonic lethal, while heterozygotes had a higher proclivity to develop tumors (4). Other studies have shown that ectopically overexpressing SWI/ SNF subunits induces senescence in cells, further supporting the notion that the correct concentration of SWI/SNF proteins is important in homeostasis and that their imbalances can lead to dire consequences (5).

\section{THE SWI/SNF COMPLEX IN CANCER}

Analysis of malignant rhabdoid tumors (MRTs) and atypical/teratoid rhabdoid tumors (AT/RT) genomes identified mutations and deletions on the SMARCB1 locus of chromosome 22, providing the first evidence of the role of the SWI/SNF complex in cancer (6). Additional studies have shown that other subunits of the SWI/SNF complex are dysregulated in cancer; a meta-analysis by Kadoch and colleagues demonstrated that the SWI/SNF complex is mutated in more than $20 \%$ of human cancers (7). A review by Hodges showed, using data from The Cancer Genome Atlas (TCGA), that SWI/SNF complex subunits are frequently mutated in human cancers, and that mutations occur in subunit-specific patterns, further supporting the importance of targeting the SWI/SNF complex as a modality of treatment (8). Previous reviews have extensively summarized the research on the SWI/ SNF complex in human cancers, demonstrating the significant role of the SWI/SNF complex in tumorigenesis, maintenance, and progression $(1,8)$. However, the mechanism of how mutations in the SWI/SNF complex lead to tumorigenesis and disease progression is still largely unknown. Deeper molecular understanding of how SWI/SNF mutations cause disease is complicated by the fact that the SWI/SNF complex is composed of up to 15 subunits that are encoded by 29 genes, which can result in 1400 different combinations of the complex assembly $(9,10)$. Given the complexity of the SWI/SNF 
complex, different mutations can result in different biological effects, highlighting the critical role of the SWI/SNF complex in regulating cell homeostasis.

This review summarizes an emerging body of data strongly suggesting that the SWI/SNF complex plays a critical role in cellular metabolism. Therefore, it is provocative to postulate that cancers and other diseases characterized by SWI/SNF genetic alterations are "metabolic diseases" that could be targeted by a new set of tools modulating the cellular metabolic status. Although the role of the SWI/SNF complex in metabolism and disease is a developing research field in humans, the role of the SWI/SNF complex in yeast (Saccharomyces cerevisiae) metabolism has been characterized to a larger extent. The SWI/SNF complex, was first studied in S. cerevisiae, but it has since been investigated in other organisms, including Drosophila melanogaster and other mammals due to the growing evidence of its fundamental role in human diseases. Since it has been extensively investigated in various organisms, the nomenclature for the SWI/SNF complex can mislead researchers in understanding the specific roles of each subunit. For clarity, this review will refer to the complex as the SWI/SNF complex and utilize the HUGO nomenclature for the subunits. Overall, this review will summarize the current body of literature demonstrating the role of the SWI/SNF complex in metabolic processes and the associated vulnerabilities in human diseases like cancer.

\section{METABOLIC FLEXIBILITY IN ENERGY HOMEOSTASIS AND DISEASE}

Metabolism can be defined as a chain of chemical processes that breaks down nutrients, enabling life in organisms. The key metabolic pathways (i.e. The Citric Acid Cycle) are highly conserved from unicellular organisms like Escherichia coli to multicellular organisms like humans (11). This high level of conservation across species of key metabolic pathways alludes to their ancient origins and their high efficiency. However, the availability of nutrients and oxygen, key components of life, are not always readily available due to a dynamic environment. Therefore, metabolic flexibility, or the ability of organisms to adapt their metabolism to fluctuating fuel availability, is critical for survival (12). Louis Pasteur demonstrated that S. cerevisiae can switch between glycolysis and mitochondrial respi- ration depending on oxygen availability in order to survive during periods of stress. Metabolic flexibility is also critical for energy homeostasis in mammals; several studies revealed that impairment of metabolic flexibility can lead to altered homeostasis (12). For example, the healthy mammalian heart relies primarily on fatty acid oxidation as its source of fuel, but has the ability to rapidly switch substrates in order to adapt to physiological changes (i.e. exercise) $(13,14)$. However, in both mouse and rat models of type 1 and type 2 diabetes, researchers demonstrated that the diabetic heart is almost exclusively constrained to the use of fatty acid oxidation for its source of ATP $(15,16)$. Studies in humans showed that fatty acid oxidation is increased in individuals with type 1 diabetes, supporting the findings in animals models (17).

Cancer is another example of a disease with $a b-$ normal metabolism. Tumor cells are hyper-proliferative cells that require a constant influx of energy and nutrients in order to maintain their rapid growth and dissemination (18). Therefore, studies have shown that tumor cells can rewire their metabolism to adapt to their environment and needs. Otto Warburg first described one of these metabolic adaptations utilized by tumor cells in his observation that tumor cells used "aerobic" glycolysis or metabolized glucose even in the presence of oxygen (19). This phenomenon is now termed the "Warburg effect" or "aerobic glycolysis," and it is seen in various cancers. Cancer metabolism is often mistakenly associated with Warburg effect, or aerobic glycolysis. Although some tumor cells exhibit Warburg effect, this is not true in all cases (20). In 2011, Hanahan \& Weinberg included deregulation of metabolism as a new hallmark of cancer cells (21). Indeed, an enormous amount of evidences following Warburg's findings sustained how cancer cells increase their dependency from glucose. For example, oncogenes like RAS or MYC can fuel the glycolytic pathways of tumoral cells (22).

On the contrary, emerging evidences revealed that at least two differently metabolically regulated subpopulations are present in tumors: one subpopulation reflecting the metabolic changes as described by Warburg's effect, and a second population characterized by dependency on other metabolic pathways, such as oxidative phosphorylation (OXPHOS) (23). These premises highlight the metabolic plasticity of cancer cells in terms of adaptation and survival to different environmental or cell autonomous events. In this review, we 
will summarize the different metabolic roles of the SWI/SNF complex described in recent studies.

\section{THE SWI/SNF COMPLEX REGULATES CARBON FLEXIBILITY}

The genes encoding the different subunits of the complex were first revealed in screens for mating type switching and sucrose metabolism in S. cerevisiae, providing the first evidence of the involvement of the SWI/SNF complex in carbohydrate metabolism. This genetic screen identified five new sucrose non-fermenting (Snf) alleles, Snf2, Snf3, Snf4, Snf5, and Snf6 in addition to Snf1 as positive regulators of $S U C 2$, a member of the SUC gene family that encodes invertase to hydrolyze extracellular sucrose into glucose and fructose (24). Snf2 and Snf5 were later confirmed as members of the yeast SWI/SNF complex. Both Snf2 (BRG/BRM) and Snf5 (SMARCB1) also have mammalian homologs that are implicated in human diseases.

Hypoxia is one of the most studied environmental stresses impacting cellular metabolism and general homeostasis, depleting ATP reserves and altering carbohydrate metabolism (25). Interestingly, a study by Burgain and colleagues demonstrated that Candida albicans (C. albicans) with Snf5 (also called SMARCB1) mutations cannot grow on alternative carbon sources in hypoxia. However, the mutants can grow normally on fermentable carbohydrates like glucose and fructose (26). The authors determined that Snf5 is required for survival in hypoxia via regulation of carbon flexibility, ultimately concluding that the SWI/SNF complex is a master regulator that connects the oxygen-sensing machinery with carbon utilization in C. albicans. The metabolic phenotypes seen in SWI/SNF mutants in S. cerevisiae previously described suggest that the role of SWI/SNF is to maintain overall carbohydrates homeostasis by regulating metabolic pathways. As eluded in S. cerevisiae and C. albicans, the SWI/SNF complex probably plays a critical role in carbon flexibility in humans as well, and the loss of this flexibility leads to various metabolic vulnerabilities that can be exploited therapeutically as it will be summarized in the following. Depending on the availability of nutrients and oxygen in the tumor microenvironment, the loss of the SWI/SNF complex can possibly manifest into different metabolic phenotypes in a context-dependent manner such as tissue type, timing, and energy requirements.

\section{THE SWI/SNF COMPLEX IN OXPHOS}

Recent reports suggest that several cancers with inactivating mutations of the SWI/SNF complex subunits are dependent on OXPHOS. An analysis of RNA-sequencing data from The Cancer Genome Atlas (TCGA) project of human lung adenocarcinoma tumors revealed that the most enriched pathway in tumors with SMARCA4 and ARID1A mutations is the oxidative phosphorylation pathway (27). In this study, Lissanu and colleagues found that metabolic genes, such as peroxisome proliferator-activated receptor-gamma coactivator (PGC1-a), mitochondrial ATP synthase F0 complex subunit ATP5L, and genes involved in oxidative stress response, including glutathione S-transferase GSTO7 and GSTO1, were enriched in lung adenocarcinoma tumors with SMARCA4 and ARID1A mutations. PGC1-a is known to drive mitochondrial respiration and biogenesis, and shRNA mediated knockdown of PGC1-a in a SMARCA4-deficient lung cancer cell line was significantly detrimental to their growth in colony formation assays whereas, re-expression of SMARCA4 reversed the phenotype, confirming its dependency on SMARCA4 (27).

In the same study, it was demonstrated that tumors derived from a KPS (Kras ${ }^{\text {LLLG12DNWT, p53 }} 3^{\mathrm{flff}}$, Smarca4 $\left.4^{f / \mid f l}\right)$ Smarca4-deficient genetically engineered mouse model displayed enriched OXPHOS based on gene enrichment analysis (GSEA) compared to tumors derived from the KP (Kras ${ }^{\text {LLLG12D/WT, }}$ $\left.p 53^{f / f l}\right)$ mouse model, providing further evidence of the role of Smarca4 in regulating OXPHOS (27). They also demonstrated, using a novel inhibitor of mitochondrial complex I (IACS-010759), that cancer cell lines and xenografts with inactivating mutations in a subunit of the SWI/SNF complex (either SMARCA4 or ARID1A) were more sensitive to inhibition of OXPHOS in comparison to their wild-type counterparts. These results highlight the fact that the loss of SWI/SNF complex members confers a unique metabolic vulnerability that can be exploited to treat cancers with these mutations.

\section{THE SWI/SNF COMPLEX REGULATES FATTY ACID OXIDATION/LIPID METABOLISM}

Interestingly, PGC1-a is implicated in the interaction with the SWI/SNF complex by independent studies. A genome-wide coactivation screen in mouse livers identified BAF60a (SMARCD1), a member of the 
SWI/SNF complex, to have nearly overlapping activity to PGC-1 a (28). Further investigation revealed that BAF60a increases the mitochondrial DNA content, the expression levels of respiratory complex proteins, and fatty acid $\beta$-oxidation genes. $\mathrm{Li}$ and colleagues showed that BAF60a and PGC-1a in teracted in the cell, and co-immunoprecipitation (IP) studies revealed that PGC-1a associated with BRG1 and BAF53a, two other members of the SWI/ SNF complex, in addition to BAF60a. Pharmacological activation of peroxisome proliferator-activated receptor a (PPARa), which interacts with PGC-1 a to regulate fatty acid oxidation, amplified the activation of fatty acid $\beta$-oxidation genes by BAF60a.

RNAi knockdown studies in the liver of fasted mice showed that the livers of BAF60a-deficient mice had impaired fatty acid oxidation with larger fat droplets, based on Oil Red O staining, increased free fatty acids, and a 2-fold increase in triglycerides (TG) content (28). Chromatin immunoprecipitation (ChIP)-qPCR analysis also revealed that BAF60a recruitment to the FAO promoters during fasting in mice are significantly compromised in PGC1-a deficient murine livers, suggesting that PGC1- $\alpha$ is required for mediating the recruitment of BAF60a to the FAO gene promoters in liver (28). Similarly, Meng and colleagues showed in a mouse model with conditional BAF60a deficiency in the liver that BAF60a plays a role in mediating plasma cholesterol levels in response to diet. Also, BAF60a inactivation protected mice from diet-induced hypercholesterolemia and atherosclerosis (29). Together, the results of this study provided evidence of the role of the SWI/SNF complex in promoting fatty acid oxidation and hepatic lipid metabolism, particularly during metabolic stress such as fasting, via the PGC-1a/PPARa molecular pathway.

\section{THE SWI/SNF COMPLEX IN GLUCOSE SENSING AND METABOLISM}

Lissanu and colleagues also observed that removal of glucose from the growth media augmented the effects of OXPHOS inhibition in lung tumor cell lines deficient in SMARCA4, and reconstitution of SMARCA4 was able to prevent cell death by glucose deprivation in A549 and H1299 cells. (27) These results lead the investigators to speculate that SMARCA4 might have a broader role in energy-deprivation-induced stress. This result is reminiscent of studies in S. cerevisiae and C. albi- cans that demonstrated the role of the SWI/SNF complex in regulating carbohydrate metabolism. In these studies, investigators showed that opportunistic organisms like yeast and fungus with SWI/ SNF inactivating mutations had impaired carbohydrate metabolism and flexibility $(24,26,30,31)$.

Meng and colleagues also demonstrated that BAF60C (SMARCD3) is involved in coordinating muscle adaptation to high endurance exercise through the PGC1-a pathway by regulating glycolytic and oxidative metabolism (32). Specifically, they showed that BAF60C is required in skeletal muscle for maintaining glycolytic capacity to improve glucose homeostasis through the Deptor-mediated AKT pathway (33). Another study by Meng and colleagues also showed that the SWI/SNF complex is an important glucose sensor in myocytes through the Baf60c-Deptor-AKT signaling axis (34). Together, these studies suggest that human cancers with inactivating mutations of the SWI/SNF complex also lack carbohydrate flexibility and might be exceptionally vulnerable to glucose deprivation.

\section{SWI/SNF MUTATIONS AND ABNORMAL GLYCOGEN ACCUMULATION}

Glycogen turnover is a critical part of cellular metabolic adaptation to environmental stress caused by activities such as high-intensity exercise (35). The breakdown of glycogen releases glucose-1-phosphate that is converted into glucose-6-phosphate, which then enters glycolysis or the pentose phosphate pathway (PPP) (36). Considering that several malignancies utilize glycolysis to support rapid proliferation, glycogen turnover may be a key player in their pathogenesis. If glucose-6-phosophate enters PPP, it contributes to several essential metabolic activities including the generation of nucleotides, $\mathrm{NADPH}$, amino acid synthesis, lipid synthesis, and reactive oxygen species (ROS) scavenging molecules that help maintain cell homeostasis and growth (37). The presence of abnormal glycogen metabolism has been described in tumors, and its potential importance in cancer pathophysiology is acknowledged, although it is still not clearly understood (38). Studies have reported that glycogen storage is inversely correlated with tumor proliferation, suggesting that it may be utilized as a fuel to support tumor growth (39). The accumulation of glycogen in both cancer and non-cancer cells has been 
reported in hypoxic conditions as well (40-44). A study by Favoro et al. demonstrated that glycogen levels are increased in the hypoxic areas of tumors and are induced by bevacizumab, an inhibitor of angiogenesis (37). This study reported that upon hypoxia $\left(0.1 \% \mathrm{O}_{2}\right)$ in vitro there was an initial rapid accumulation of glycogen (24 hours of hypoxia) followed by a decline (72 hours of hypoxia) in U87 (glioblastoma), MCF-7 (breast), and HCT116 (colon) cancer cell lines,. Accordingly, GYS1 (glycogen synthase stores glycogen) increased initially, but gradually declined after prolonged hypoxia (72 hours), while PYGL (glycogen phosphorylase breaks down glycogen in the cytosol) gradually increased and peaked at 72 hours of hypoxia. The depletion of PYGL led to a decrease in proliferation, induced p53-dependent senescence, and increased glycogen and ROS, suggesting that glycogen breakdown is required for free radical protection and growth in hypoxia. This study also showed that glucose utilization through glycogen is necessary for optimum expansion of proliferating cancer cells (37). Cumulatively, these findings highlight the importance of glycogen turnover in cancer pathogenesis and progression, especially in the context of hypoxia.

In particular, "clear cell carcinomas" derive their name from the presence of large, clear vacuoles in their cytoplasm that result from the extraction of glycogen during histological processing. It is noteworthy that cancers of the clear cell sub-type are characterized by aberrations in the SWI/SNF complex. A study by Steinberg and colleagues investigated the glucose metabolism in different clear cell cancers and showed that cancers with the clear cell phenotype have increased levels of glycogen, glucose-6-phosphate, andglycolytic activity, and a reduced gluconeogenesis (45). Another study by Wang and colleagues demonstrated that CARM1, an arginine methyltransferase, methylates BAF155, a core member of the SWI/SNF complex (46). Interestingly, a separate study showed that CARM1 is required for glycogen gene expression program in skeletal muscles (47). Together, these observations lead Delattre and colleagues to postulate that alterations in the SWI/SNF complex "induce excessive glycogen accumulation as a consequence of abnormal carbohydrate metabolism" (48). Delattre further speculated that the clear cell phenotype might be indicative of SWI/SNF aberrations in other rare tumors with this histotype that have not yet been characterized in large-scale genomic studies.
Interestingly, this phenomenon is well described in $S$. cerevisiae yeast, providing potential insight into the role of SWI/SNF in metabolic regulation of glycogen metabolism. In the presence of abundant glucose, S. cerevisiae will generate ATP via glycolysis. However, when glucose is exhausted, ATP levels decrease and AMP levels increase, resulting in a high AMP/ATP ratio that activates Snf1 kinase (AMP-activated protein kinase (AMPK) in humans) (49). In this state, S. cerevisiae cells grow slower and accumulate glycogen as a carbohydrate reserve before glucose, their preferred carbon source, is completely depleted. Studies demonstrated that the addition of glucose will cause $S$. cerevisiae to switch back to their preferred glycolytic metabolism (50). Mutant S. cerevisiae defective in Snf1 are known to not accumulate glycogen (51). Similarly in mammals, mice with liver specific embryonic loss of Snf5 (SMARCB1 in humans), a core member of the SWI/SNF complex, died prematurely due to severe hypoglycemia, and were not able to store glycogen properly in their cells (52). These results provide evidence that glycogen accumulation is an adaptation mechanism to stress such as nutrient deprivation that is likely modulated by the SWI/ SNF complex.

\section{THE SWI/SNF COMPLEX IN GLUTATHIONE METABOLISM/ OXIDATIVE STRESS RESPONSE}

Ogiwara and colleagues demonstrated that SWI/ SNF mutations in cancer caused a vulnerability to inhibition of the antioxidant glutathione (53). In this study, Ogiwara and colleagues used a drug sensitivity screen in parental HCT116 colon cancer cells and HCT116 with ARID1A-knockout to identify PRIMA-1 (APR-017), an inhibitor of glutathione, as a compound that inhibited the growth of ARID1A-deficient HCT116 colon cancer cells. They also measured a corresponding increase in ROS in ARID1A-deficient colon cancer cell lines in comparison to ARID1A-proficient colon cancer cell lines. Additionally, SLC7A11 gene expression was significantly decreased in ARID1A-deficient cancer cells, and a chromatin immunoprecipitation (ChIP) assay showed that ARID1A localized at the transcription start site of SLC7A11 in ARID1A wild-type cells. Further investigations using gas chromatography/mass spectrometry identified that cysteine was enriched greater than 2-fold in ARID1A-deficient cancer cells, and re-expression of 
SLC7A11 decreased sensitivity of ARID1A-deficient cancer cells to inhibition of glutathione. Studies in ARID1A deficient patient-derived cancer cells confirmed their findings in vitro. Altogether, these results lead Ogiwara and colleagues to conclude that ARID1A regulates the balance between basal glutathione levels and ROS through SLC7A11.

In another study, Sun and colleagues demonstrated that ARID1A promoted liver cancer initiation by increasing the levels of ROS (54). Gene set enrichment analysis (GSEA) of ARID1A overexpression versus ARID1A-deficiency in non-malignant mouse livers showed that Hnf4a, which drives the transcription of CYP450 genes, was enriched in livers with ARID1A overexpression. Similarly, ARID1A-overexpression in non-malignant livers correlated with increased enrichment of CYP450 genes, a superfamily of monooxygenases that oxidize metabolites, while ARID1A-deficiency was associated with a decrease in CYP450 genes. They demonstrated that ARID1A promoted liver cancer initiation by stimulating ROS production via the regulation of Cyp2e1 expression. In addition, they showed that suppression of Cyp2e1 after ARID1A ablation reduced oxidative stress and tumorigenesis in mice, validating that the SWI/SNF complex plays a critical role in regulating oxidative stress. This study showed that SWI/ SNF mutations had context-dependent oncogenic and tumor suppressor functions.

\section{THE SWI/SNF COMPLEX IN PROTEOTOXIC STRESS}

Few studies recently highlighted a role for the SWI/ SNF complex in regulating protein homeostasis. A study by Carugo and colleagues demonstrated that ablation of SMARCB1 in embryonic livers of mice lead to malignant rhabdoid tumors (MRT) characterized by increased endoplasmic reticulum (ER) stress to unfolded protein response (UPR) and autophagy (55). They demonstrated, using novel embryonic mosaic mouse models of MRT, that SMARCB1-deficient tumors are sensitive to combinatorial inhibition of the proteasome machinery and autophagy pathways using bortezomib/ ixazomib and Chloroquine, respectively. They also showed that p53 and MYC mRNA were enriched in SMARCB1-deficient tumors, and they went on to demonstrate that inhibition of the proteostatic arm of p53 leads to tumor regression in the context of SMARCB1-deficiency. Similarly, pancreatic ad- enocarcinomas characterized by downregulation of SMARCB1 and other members of the SWI/SNF complex resulted in undifferentiated/sarcoma-like transformation with aggressive behavior $(56,57)$. Upon SMARCB1 downregulation, pancreatic cancer cells exhibited a defective regulation of proteostasis; such disruption of the proteostatic equilibrium leads to specific vulnerabilities of mesenchymal pancreatic cancer cells that can be exploited therapeutically with clinically available drugs $(56,57)$. These results demonstrated yet another way in which SWI/SNF mutations lead to metabolic abnormalities and vulnerabilities in cancer that can be targeted therapeutically. Altogether, these studies support prior reports showing how mutations in the SWI/SNF complex initially play tumor suppressor roles in tumor initiation but have additional functions in tumor maintenance in a context-dependent manner.

\section{THERAPEUTIC IMPLICATIONS OF SWI/SNF ALTERATIONS IN CANCER}

The aforementioned molecular mechanisms and vulnerabilities following alterations of members of the SWI/SNF complex open potential new opportunities for clinical translation (table I). In particular, targeting of metabolic pathways with highly selective compounds might be a feasible and impactful therapeutic intervention. For example, Carugo and colleagues demonstrated that ixazomib and bortezomib (FDA approved drugs), specifically targeting proteasome machinery, strongly affect cell vitality and growth of SMARCB1 deleted tumors; this pharmacological setting is now tested in clinical trials for SMARCB1 deleted urological malignancies (NCT03587662). Similarly, Lissanu and colleagues showed that SMARCA4 mutated cells are more sensitive to OXPHOS inhibition and inhibitors of OXPHOS are being tested on numerous cancer types (NCT03291938). Finally, ovarian cancer cells with inactivation for ARID1A have been described to be more dependent on glutamine metabolism rendering this particular tumor more sensitive to GLS1 inhibition via CB-839, a clinically available glutaminase inhibitor (58). Taken together, these literature data strongly encourage future research on specific metabolic vulnerabilities of SWI/SNF dysfunctional tumors, whereas metabolic targeted therapy can be applied as a single treatment regimen or in combination with cytotoxic chemotherapy. 


\begin{tabular}{|c|c|c|c|c|}
\hline SUBUNIT & SPECIES & ORGAN/DISEASE & VULNERABILITY & REFERENCE \\
\hline $\begin{array}{l}\text { Snf2 (hBRM) /Snf5 } \\
\text { (Smarcb1) }\end{array}$ & $\begin{array}{l}\text { Saccharomyces } \\
\text { cerevisiae }\end{array}$ & N/A & $\begin{array}{l}\text { glucose/carbohydrate } \\
\text { metabolism }\end{array}$ & $\begin{array}{l}\text { Neigeborn and } \\
\text { Carlson } 1984\end{array}$ \\
\hline Snf5 (Smarcb1) & Candida albicans & N/A & $\begin{array}{l}\text { glucose/carbohydrate } \\
\text { metabolism in hypoxia }\end{array}$ & Burgain 2019 \\
\hline SMARCA4/ARID1A & Homo sapiens & $\begin{array}{l}\text { lung } \\
\text { adenocarcinoma }\end{array}$ & OXPHOS & Lissanu 2018 \\
\hline Baf60a & Mus musculus & liver & fatty acid oxidation & Li 2008 \\
\hline Baf60a & Mus musculus & liver & plasma cholesterol & Meng 2015 \\
\hline SMARCA4 & Homo sapiens & $\begin{array}{l}\text { lung } \\
\text { adenocarcinoma }\end{array}$ & glucose metabolism & Lissanu 2018 \\
\hline $\begin{array}{l}\text { Baf60c (encoded by } \\
\text { SMARCD3) }\end{array}$ & Mus musculus & muscle & glucose homeostasis & Meng 2013 \\
\hline Baf155 & Homo sapiens & breast cancer cells & glycogen metabolism & Wang 2014 \\
\hline ARID1A & $\begin{array}{l}\text { Homo sapiens, } \\
\text { Mus musculus }\end{array}$ & $\begin{array}{l}\text { ovarian clear cell } \\
\text { carcinomas, non- } \\
\text { malignant liver }\end{array}$ & $\begin{array}{l}\text { glutathione } \\
\text { metabolism/oxidative } \\
\text { stress response }\end{array}$ & $\begin{array}{l}\text { Sun 2017, Ogiwara } \\
2019\end{array}$ \\
\hline SMARCB1, ARID1A & $\begin{array}{l}\text { Homo sapiens, } \\
\text { Mus musculus }\end{array}$ & $\begin{array}{l}\text { malignant } \\
\text { rhabdoid tumors, } \\
\text { pancreatic cancer }\end{array}$ & proteotoxic stress & $\begin{array}{l}\text { Carugo 2019, } \\
\text { Tomihara } 2021\end{array}$ \\
\hline
\end{tabular}

Table III. SWI/SNF mutations and metabolic vulnerabilities.

\section{CONCLUSIONS}

We have summarized in this review how SWI/SNF mutations in diseases like cancer lead to metabolic dysregulations that could be targeted therapeutically. SMARCB1 and other SWI/SNF subunits have been shown to be tumor suppressors in cancer. However, we highlighted how mutations in the SWI/ SNF complex subunits can have context-dependent functions in tumor initiation, tumor progression and maintenance. Such mechanisms of tumor progression appear to be unique metabolic adaptations that can be targeted for the treatment of cancer. Paralleling molecular and functional studies in $S$.

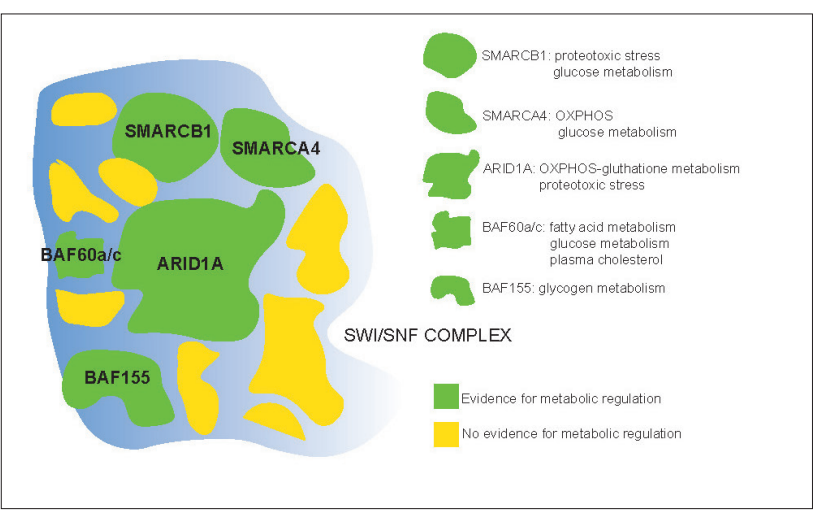

Figure 2. Schematic of SWI/SNF subunits that have known metabolic regulatory roles cerevisiae with human pathology demonstrates the importance of the SWI/SNF complex in regulating metabolic adaptations that are critical for survival in an environment that has fluctuating resources like oxygen and nutrients (figure 2). Cancer cells due to their abnormal proliferative capacity also have a high energy demand that requires metabolic adaptation mechanisms to support their rapid expansion. Based on the literature we have summarized in this review, the SWI/SNF complex appears to play a multifaceted role in regulating metabolism from glucose to protein homeostasis in higher eukaryotes like humans in addition to $S$. cerevisiae, and mutations in the complex lead to metabolic dysregulations that enable rapidly dividing cells to survive in microenvironments with limited or depleting resources. However, as all of these studies have demonstrated, these metabolic adaptations, which are initially beneficial to proliferating tumor cells, are also the Achilles' heel of cancers with SWI/SNF mutations, as Gorrini described it (59). Therefore, we conclude that cancers and other diseases that are characterized by SWI/ SNF mutations are metabolic diseases, and future therapies should focus on targeting the unique metabolic vulnerabilities associated with SWI/SNF complex mutations. 


\section{ETHICS}

\section{Fundings}

GG was supported by the Barbara Massie Memorial Fund, the MDACC Moonshot FIT Program, the Bruce Krier Endowment Fund, and the Lyda Hill Foundation.

\section{Conflict of interests}

The authors have declared no conflict of interests.

\section{Avalability of data and material}

No datasets were generated or analyzed during the current study.

\section{REFERENCES}

1. Wilson BG, Roberts CW. SWI/SNF nucleosome remodellers and cancer. Nat Rev Cancer 2011;11(7):481-92.

2. Saha A, Wittmeyer J, Cairns BR. Chromatin remodelling: the industrial revolution of DNA around histones. Nat Rev Mol Cell Biol 2006;7(6):437-47.

3. Alessio N, Squillaro T, Cipollaro M, Bagella L, Giordano A, Galderisi U. The BRG1 ATPase of chromatin remodeling complexes is involved in modulation of mesenchymal stem cell senescence through RB-P53 pathways. Oncogene 2010;29(40):5452-63.

4. Bultman S, Gebuhr T, Yee D, et al. A Brg1 null mutation in the mouse reveals functional differences among mammalian SWI/SNF complexes. Mole Cell 2000;6(6):1287-95.

5. Napolitano MA, Cipollaro M, Cascino A, Melone MA, Giordano A, Galderisi U. Brg1 chromatin remodeling factor is involved in cell growth arrest, apoptosis and senescence of rat mesenchymal stem cells. J Cell Sci 2007;120(16):2904-11.

6. Versteege I, Sevenet N, Lange J, et al. Truncating mutations of hSNF5/INI1 in aggressive paediatric cancer. Nature 1998;394(6689):203-6.

7. Kadoch C, Crabtree GR. Mammalian SWI/SNF chromatin remodeling complexes and cancer: Mechanistic insights gained from human genomics. Sci adv 2015;1(5):e1500447.

8. Hodges C, Kirkland JG, Crabtree GR. The many roles of BAF (mSWI/SNF) and PBAF complexes in cancer. Cold Spring Harbor perspectives in

\section{Authors' contribution}

Writing including the original draft, review, and editing was performed by MS, LP, and GG. Writing including review and editing was performed by MS, LP, and GG. Figures preparation was performed by MS and LP. This work was supervised by GG and AS. All authors have read and agreed to the published version of the manuscript.

\section{Ethical approval}

N/A

\section{Consent to participate \\ N/A}

9. Kadoch C, Hargreaves DC, Hodges C, et al. Proteomic and bioinformatic analysis of mammalian SWI/SNF complexes identifies extensive roles in human malignancy. Nature Gen 2013;45(6):592-601.

10. Wang W, Côté J, Xue Y, et al. Purification and biochemical heterogeneity of the mammalian SWI-SNF complex. The EMBO J 1996;15(19):5370-82.

11. Peregrín-Alvarez JM, Sanford C, Parkinson J. The conservation and evolutionary modularity of metabolism. Genome Biol 2009;10(6):R63-R.

12. Goodpaster BH, Sparks LM. Metabolic Flexibility in Health and Disease. Cell metabol 2017;25(5):1027-36.

13. Duncan JG. Peroxisome proliferator activated receptor-alpha (PPARa) and PPAR gamma coactivator-1alpha (PGC-1a) regulation of cardiac metabolism in diabetes. Pediatr Cardiol 2011;32(3):323-8.

14. Neely J, Rovetto Ma, Oram J. Myocardial utilization of carbohydrate and lipids. Progress in cardiovascular diseases 1972;15(3):289-329.

15. Belke DD, Larsen TS, Gibbs EM, Severson DL. Altered metabolism causes cardiac dysfunction in perfused hearts from diabetic ( $\mathrm{db} /$ db) mice. Am J Physiol-Endocrinol Metabol 2000;279(5):E1104-E13.

16. Gamble J, Lopaschuk GD. Glycolysis and glucose oxidation during reperfusion of ischemic 
hearts from diabetic rats. Biochimica et Biophysica Acta (BBA)-Molecular Basis of Disease 1994;1225(2):191-9.

17. Herrero P, Peterson LR, McGill JB, et al. Increased myocardial fatty acid metabolism in patients with type 1 diabetes mellitus. J Am College Cardiol 2006;47(3):598-604.

18. Zhu J, Thompson CB. Metabolic regulation of cell growth and proliferation. Nat Rev Mol cell biol 2019;20(7):436-50.

19. Warburg $\mathrm{O}$. The metabolism of carcinoma cells. J Canc Res 1925;9(1):148-63.

20. Semenza GL. Tumor metabolism: cancer cells give and take lactate. J clin investigation 2008;118(12):3835-7.

21. Hanahan D, Weinberg RA. Hallmarks of cancer: the next generation. Cell 2011;144(5):646-74.

22. DeBerardinis RJ, Lum JJ, Hatzivassiliou G, Thompson CB. The biology of cancer: metabolic reprogramming fuels cell growth and proliferation. Cell metabolism 2008;7(1):11-20.

23. Viale A, Pettazzoni P, Lyssiotis CA, et al. Oncogene ablation-resistant pancreatic cancer cells depend on mitochondrial function. Nature 2014;514(7524):628-32.

24. Neigeborn L, Carlson M. Genes affecting the regulation of SUC2 gene expression by glucose repression in Saccharomyces cerevisiae. Genetics 1984;108(4):845-58.

25. Semenza GL. Regulation of mammalian O2 homeostasis by hypoxia-inducible factor 1 . Annual review of cell and developmental biology 1999;15(1):551-78.

26. Burgain A, Pic E, Markey L, Tebbji F, Kumamoto CA, Sellam A. A novel genetic circuitry governing hypoxic metabolic flexibility, commensalism and virulence in the fungal pathogen Candida albicans. PLoS pathogens 2019;15(12):e1007823.

27. Lissanu Deribe $Y$, Sun $Y$, Terranova C, Khan F, Martinez-Ledesma J, Gay J, et al. Mutations in the SWI/SNF complex induce a targetable dependence on oxidative phosphorylation in lung cancer. Nature med 2018;24(7):1047-57.

28. Li S, Liu C, Li N, et al. Genome-wide coactivation analysis of PGC-1a identifies BAF60a as a regulator of hepatic lipid metabolism. Cell metabolism 2008;8(2):105-17.

29. Meng ZX, Wang L, Chang L, et al. A Diet-Sensitive BAF60a-Mediated Pathway Links Hepatic Bile Acid Metabolism to Cholesterol Absorption and Atherosclerosis. Cell reports
2015;13(8):1658-69.

30. Abrams E, Neigeborn L, Carlson M. Molecular analysis of SNF2 and SNF5, genes required for expression of glucose-repressible genes in Saccharomyces cerevisiae. Molecular and cellular biology 1986;6(11):3643-51.

31. Carlson M, Osmond BC, Botstein D. Mutants of yeast defective in sucrose utilization. Genetics 1981;98(1):25-40.

32. Meng ZX, Tao W, Sun J, Wang Q, Mi L, Lin JD. Uncoupling Exercise Bioenergetics From Systemic Metabolic Homeostasis by Conditional Inactivation of Baf60 in Skeletal Muscle. Diabetes 2018;67(1):85-97.

33. Meng ZX, Li S, Wang L, et al. Baf60c drives glycolytic metabolism in the muscle and improves systemic glucose homeostasis through Deptor-mediated Akt activation. Nat Med 2013;19(5):640-5.

34. Meng ZX, Gong J, Chen Z, et al. Glucose Sensing by Skeletal Myocytes Couples Nutrient Signaling to Systemic Homeostasis. Mol cell 2017;66(3):332-44.e4.

35. Hearris MA, Hammond KM, Fell JM, Morton JP. Regulation of Muscle Glycogen Metabolism during Exercise: Implications for Endurance Performance and Training Adaptations. Nutrients 2018;10(3):298.

36. Roach PJ, Depaoli-Roach AA, Hurley TD, Tagliabracci VS. Glycogen and its metabolism: some new developments and old themes. Biochem J 2012;441(3):763-87.

37. Favaro E, Bensaad K, Chong Mei G, et al. Glucose Utilization via Glycogen Phosphorylase Sustains Proliferation and Prevents Premature Senescence in Cancer Cells. Cell Metab 2012;16(6):751-64.

38. Zois CE, Favaro E, Harris AL. Glycogen metabolism in cancer. Biochem Pharmacol 2014;92(1):3-11.

39. Rousset M, Zweibaum A, Fogh J. Presence of glycogen and growth-related variations in 58 cultured human tumor cell lines of various tissue origins. Cancer res 1981;41(3):1165.

40. Mamedova L, Shneyvays V, Katz A, Shainberg A. Mechanism of glycogen supercompensation in rat skeletal muscle cultures. Mol cell biochem 2003;250(1-2):11-9.

41. Pelletier J, Bellot G, Gounon P, Lacas-Gervais S, Pouysségur J, Mazure NM. Glycogen Synthesis is Induced in Hypoxia by the Hypoxia-Inducible Factor and Promotes Cancer Cell Survival. Frontiers Oncol 2012;2:18-. 
42. Pescador N, Villar D, Cifuentes D, et al. Hypoxia Promotes Glycogen Accumulation through Hypoxia Inducible Factor (HIF)-Mediated Induction of Glycogen Synthase 1 (Induction of GYS1 by Hypoxia). PloS one 2010;5(3):e9644.

43. Shen GM, Zhang FL, Liu XL, Zhang JW. Hypoxia-inducible factor 1-mediated regulation of PPP1R3C promotes glycogen accumulation in human MCF-7 cells under hypoxia. FEBS letters 2010;584(20):4366-72.

44. Vigoda A, Mamedova L, Shneyvays V, Katz A, Shainberg A. Glycogen metabolism in rat heart muscle cultures after hypoxia. Mol cell biochem 2003;254(1-2):311-8.

45. Steinberg P, Storkel S, Oesch F, Thoenes W. Carbohydrate metabolism in human renal clear cell carcinomas. Lab Invest 1992;67(4):506-11.

46. Wang L, Zhao Z, Meyer MB, et al. CARM1 methylates chromatin remodeling factor BAF155 to enhance tumor progression and metastasis. Cancer cell 2014;25(1):21-36.

47. Wang SC, Dowhan DH, Eriksson NA, Muscat GE. CARM1/PRMT4 is necessary for the glycogen gene expression programme in skeletal muscle cells. Biochem J 2012;444(2):323-31.

48. Masliah-Planchon J, Bieche I, Guinebretiere JM, Bourdeaut F, Delattre O. SWI/SNF chromatin remodeling and human malignancies. Ann rev pathol 2015;10:145-71.

49. Wilson WA, Hawley SA, Hardie DG. Glucose repression/derepression in budding yeast: SNF1 protein kinase is activated by phosphorylation under derepressing conditions, and this correlates with a high AMP:ATP ratio. Current Biol 1996;6(11):1426-34

50. Rolland F, Winderickx J, Thevelein JM. Glucose-sensing and -signalling mechanisms in yeast. FEMS yeast research 2002;2(2):183-201. 51. Hardy TA, Huang D, Roach PJ. Interactions between CAMP-dependent and SNF1 protein kinases in the control of glycogen accumulation in Saccharomyces cerevisiae. J biol chem 1994;269(45):27907-13.

52. Gresh L, Bourachot B, Reimann A, et al. The SWI/SNF chromatin-remodeling complex subunit SNF5 is essential for hepatocyte differentiation. The EMBO j 2005;24(18):3313-24.

53. Ogiwara H, Takahashi K, Sasaki M, et al. Targeting the Vulnerability of Glutathione Metabolism in ARID1A-Deficient Cancers. Cancer cell 2019;35(2):177-90.e8.

54. Sun X, Wang SC, Wei Y, et al. Arid1a has context-dependent oncogenic and tumor suppressor functions in liver cancer. Cancer cell 2017;32(5):574-89.e6.

55. Carugo A, Minelli R, Sapio L, et al. p53 Is a Master Regulator of Proteostasis in SMARCB1-Deficient Malignant Rhabdoid Tumors. Cancer cell 2019;35(2):204-20.e9.

56. Tomihara $\mathrm{H}$, Carbone $\mathrm{F}$, Perelli $\mathrm{L}$, et al. Loss of ARID1A Promotes Epithelial-Mesenchymal Transition and Sensitizes Pancreatic Tumors to Proteotoxic Stress. Cancer res 2021;81(2):332-43.

57. Genovese G, Carugo A, Tepper J, et al. Synthetic vulnerabilities of mesenchymal subpopulations in pancreatic cancer. Nature 2017;542(7641):362-6.

58. Wu S, Fukumoto T, Lin J, et al. Targeting glutamine dependence through GLS1 inhibition suppresses ARID1A-inactivated clear cell ovarian carcinoma. Nat Canc 2021;2(2):189-200.

59. Gorrini C, Mak TW. Glutathione Metabolism: An Achilles' Heel of ARID1A-Deficient Tumors. Canc cell 2019;35(2):161-3. 